\title{
THE USE OF A GLOBULIN SUBSTANCE DERIVED FROM BEEF PLASMA AS A LOCAL HEMOSTATIC IN HEMOPHILIA ${ }^{\mathbf{1 , 2}}$
}

\author{
BY FREDERICK J. POHLE AND F. H. L. TAYLOR \\ (From the Thorndike Memorial Laboratory, Second and Fourth Medical Services (Harvard), \\ Boston City Hospital, and the Department of Medicine, \\ Harvard Medical School, Boston)
}

(Received for publication June 2, 1938)

Previous studies $(1,2)$ indicate that the defect in coagulation of the blood in hemophilia resides in a plasma globulin fraction. This globulin fraction, prepared by isoelectric precipitation at $\mathrm{pH}$ 6.0 from citrated cellular-free normal human plasma, possesses marked clot accelerating properties for hemophilic blood in vitro and in vivo. This material, called "globulin substance" because of the lack of definite knowledge of its nature, when prepared in equal quantity from hemophilic plasma possesses only slight clot promoting power. Patek and Taylor (1) prepared globulin substance from certain animal plasmas and showed in preliminary studies that it possessed clot promoting powers for hemophilic blood in vitro. The present communication reports in detail certain properties of globulin substance prepared from beef plasma and particularly its uses as a local hemostatic in hemophilia.

\section{METHODS}

Coagulation time. The method by which the coagulation time of venous blood was determined has been fully described elsewhere (2). Normal values for human blood obtained by this method were from 6 to $12 \mathrm{~min}$ utes. The coagulation time for beef blood determined by the same method was from 3 to 5 minutes.

Preparation of globulin substance from beef plasma. The method of preparation of globulin substance from beef plasma was similar to that described previously for human plasma (2). Blood was collected directly from the jugular veins of steers, after exposure of the vessel by a superficial incision. Care was taken to avoid contamination with "tissue juice." The blood was citrated to a final concentration of $\mathbf{0 . 2 5}$ per cent sodium citrate. These procedures were carried out in a packing house on stunned animals. The blood was centrifuged at 2,000

1 This study was aided, in part, by a grant given in honor of Francis Weld Peabody by the Ella Sachs Plotz Foundation.

2 The results of this investigation were presented in part at the meeting of the American Society for Clinical Investigation, Atlantic City, New Jersey, May 2, 1938. r.p.m. for 45 minutes $^{3}$ and the plasma filtered through two thicknesses of Number 2 Whatman filter paper using Buchner filtration and finally rendered free of cellular material by passage through a Berkefeld $\mathrm{V}$ filter. The Berkefeld filtrate was diluted with 10 volumes of distilled water and the globulin substance precipitated by acidifying to $\mathrm{pH} 6.0$ by the addition of 1 per cent acetic acid. The acid was added slowly with constant stirring and a final $\mathrm{pH}$ adjustment made with the aid of the quinhydrone electrode. Approximately $16 \mathrm{cc}$. of 1 per cent acetic acid are required to reduce the $\mathrm{pH}$ of $100 \mathrm{cc}$. of diluted beef plasma to 6.0 .

The precipitate of globulin substance was removed by centrifuging at 2,000 r.p.m. for 20 minutes and the supernatant liquid discarded. The precipitate was removed without dilution to a vacuum dessicator and dried. It was then finely ground and stored in a dessicator over calcium chloride. Approximately $750 \mathrm{mgm}$. of the final dried product were obtained from each $100 \mathrm{cc}$. of plasma.

Test for potency of beef globulin substance. The clot accelerating properties of a saline suspension of globulin substance were tested in vitro for hemophilic blood as previously described (2). In all experiments the dried material was suspended in the same volume of 0.85 per cent sodium chloride solution as the volume of plasma from which it was derived and centrifuged free of particulate matter. As a control for the saline suspension of globulin substance, varying amounts of isotonic saline solution were used. The addition of saline solution alone was without effect on the coagulation time of hemophilic blood.

\section{EXPERIMENTAL}

Observations on the solubility of beef globulin substance in physiological saline solution. When the dried globulin substance was suspended in the same volume of physiological saline solution as the volume of plasma from which it was derived, 68.0 per cent of the total nitrogen and 71.7 per cent of the total solids were soluble. The total nitrogen of the dried beef material was 15.3 per cent. These values were comparable to those obtained for normal human and hemophilic globulin substances (Table I). The saline suspension of

${ }^{8}$ Hematocrit values were from 41.3 per cent to 45.7 per cent cells. 
TABLE I

Observations on the solubility of beef globulin substance in physiological saline solution

\begin{tabular}{|c|c|c|c|}
\hline $\begin{array}{l}\text { Source of globulin } \\
\text { substance }\end{array}$ & $\begin{array}{c}\text { Total } \\
\text { nitrogen } \\
\text { of the } \\
\text { dried } \\
\text { material }\end{array}$ & $\begin{array}{c}\text { Total nitrogen } \\
\text { soluble in } \\
0.85 \mathrm{per} \text { cent } \\
\mathrm{NaCl}^{\text {solution }}\end{array}$ & $\begin{array}{c}\text { Solids } \\
\text { soluble in } \\
0.85 \text { per cent } \\
\text { NaCl } \\
\text { colution }\end{array}$ \\
\hline $\begin{array}{l}\text { Beef plasma .......... } \\
\text { Normal human plasma } \\
\text { Hemophilic plasma... }\end{array}$ & $\begin{array}{c}\text { per cent } \\
15.3 \\
14.0 \\
14.0\end{array}$ & $\begin{array}{c}\text { per cent } \\
68.0 \\
60.3 \\
48.4\end{array}$ & $\begin{array}{c}\text { per cent } \\
71.7 \\
67.7 \\
55.6\end{array}$ \\
\hline
\end{tabular}

globulin substance was free from particulate matter and could be passed through a Berkfeld V filter without loss of activity.

Observations on the optimum $p H$ for the precipitation of active beef globulin substance. Increasing amounts of 1 per cent acetic acid were added to each of equal portions of diluted beef plasma to produce a range of $\mathrm{pH}$ values from 6.95 to 4.95 . The precipitates from each preparation were removed, dried, and ground and the usual saline suspensions prepared. Duplicate 0.05 cc. samples of the supernatant fluid from each were then pipetted into the bottom of standard coagulation tubes and $2 \mathrm{cc}$. of hemophilic blood with a coagulation time of 70 minutes added. The globulin substance obtained by precipitation at $\mathrm{pH} 6.0$ was the most effective in reducing the coagulation time of hemophilic blood in vitro ( $\mathrm{Ta}$ ble II).

TABLE II

Effect of globulin substance prepared from beef plasma at various $\mathrm{pH}$ values on the coagulation time of hemophilic blood in vitro

\begin{tabular}{|c|c|c|c|}
\hline Plasma dilution & $\begin{array}{l}\text { Amount of } \\
\text { one per cent } \\
\text { acetic } \\
\text { acid } \\
\text { added }\end{array}$ & $\mathrm{pH}$ & $\begin{array}{l}\text { Effect on } \\
\text { coagulation } \\
\text { time* of } \\
\text { hemophilic } \\
\text { blood }\end{array}$ \\
\hline 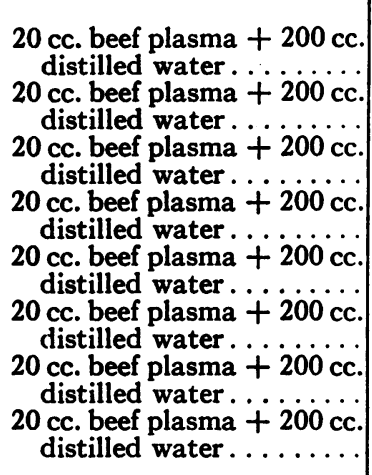 & $\begin{array}{l}1.5 \\
2.0 \\
2.5 \\
3.0 \\
4.5 \\
5.5 \\
7.5\end{array}$ & $\begin{array}{l}6.95 \\
6.60 \\
6.21 \\
6.00 \\
5.80 \\
5.45 \\
5.14 \\
4.95\end{array}$ & $\begin{array}{c}\text { minutes } \\
17.0 \\
14.5 \\
12.5 \\
6.0 \\
12.0 \\
13.5 \\
14.0 \\
15.5\end{array}$ \\
\hline
\end{tabular}

* Control coagulation time, 70 minutes.
Effect of temperature on clot accelerating properties of beef globulin substance for hemophilic blood. Portions of the usual saline suspension of beef globulin substance were subjected to different constant temperatures. At intervals, 0.05 cc. of each portion was removed and tested against 2 cc. of hemophilic blood having a coagulation time which ranged from 162 to 180 minutes. All fluids remained clear except for those kept at 48 and $52^{\circ} \mathrm{C}$. which acquired a flocculent precipitate after one hour. The effect of temperature on the clot accelerating properties of beef globulin substance is shown in Figure 1. The beef globulin substance like the human material is thermolabile but in dried form each retains its maximum potency for at least one year.

Quantitative effect of beef globulin substance on the coagulation time of hemophilic blood in vitro. Different amounts of the usual saline suspension of beef globulin substance were tested in vitro against 2 cc. blood samples from six hemophilic subjects. The clot promoting power of beef globulin substance for hemophilic blood in vitro is shown in Figure 2. There was always a marked reduction of the coagulation time with a 1:200 dilution and greater concentrations reduced it to normal and even subnormal values. Comparative studies showed that the beef globulin substance was no more potent than the plasma from which it was derived but was more potent than an equivalent amount of normal human globulin substance.

Effect of oral administration of beef globulin substance on the coagulation of hemophilic blood. One to four grams of beef globulin substance in either dried form in capsules or in saline suspension were given orally four times daily for several consecutive days to two hemophilic subjects aged 19 and 32 years. Blood coagulation times were determined frequently during the period of treatment but no acceleration of coagulation was observed. This negative effect was likewise noted following the oral administration of normal human globulin substance.

The local hemostatic properties of beef globulin substance in hemophilia. In view of the marked clot accelerating properties of beef globulin substance for hemophilic blood in the test tube it seemed reasonable to attempt its use as a local hemostatic in hemophilia. 


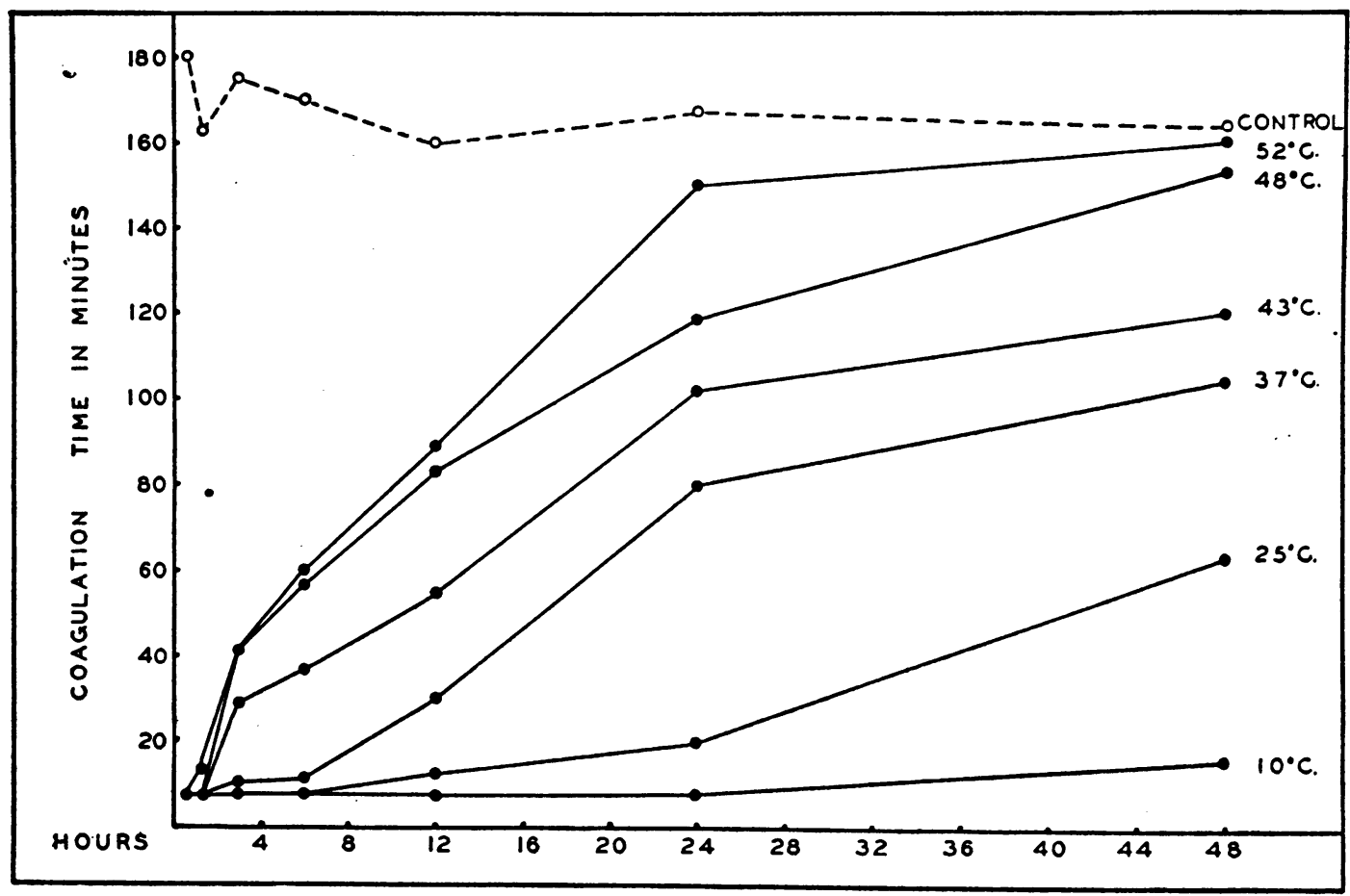

Fig. 1. Effect of Temperature on the Clot Accelerating Properties of Beef Globulin SubSTANCE FOR HEMOPHILIC BLOOD

Beef globulin substance in the form of a $d r y$ powder has been used as a local hemostatic in five adult hemophilics ranging in age from 19 to 41 years. With the exception of Case $\mathrm{V}$ these patients have been followed in this clinic for many years. All have had protracted hemorrhage from external wounds on many occasions. In each case the usual prolonged coagulation time of the blood was present at the time of these observations and was not affected by the local treatment.

Case I. (Coagulation time from 40 to 50 minutes.) On December 29, 1936 this patient sustained a laceration through the posterior scalp $4 \mathrm{~cm}$. in length and typical abnormal hemorrhage followed. The wound was sutured but continued to bleed during the next nine days. At this time the patient entered the hospital with a hemoglobin of 8.74 grams per $100 \mathrm{cc}$. of blood. There was a steady ooze of blood from the wound. During the next 24 hours hemostasis was attempted using packs containing liquid preparations of commercial thromboplastin, normal whole blood, normal plasma, and a saline suspension of normal human globulin substance without effect. All these substances were applied directly to the site of hemorrhage after removal of old clots. Beef globulin substance in the form of a dry powder placed on a saline moistened cotton pack was then applied to the wound and, as before, gentle but firm pressure main- tained with a head dressing. A firm fibrin clot formed in four minutes and hemorrhage immediately ceased. After 48 hours the sutures were removed and profuse bleeding followed which was again immediately controlled by the application of dried beef globulin substance. Five days later the wound had healed.

Four months later the right lower third molar tooth was extracted.* This operation was followed by continuous bleeding. The local application of packs containing epinephrine, thromboplastin, and moccasin snake venom was unsuccessful in controlling the hemorrhage. The application of dried beef globulin substance on a small saline moistened pack produced immediate hemostasis. The packs were removed every 24 hours to permit irrigation of the socket and each time brisk bleeding began but was immediately controlled by repacking with powdered beef globulin substance. Healing progressed normally and was complete nine days after extraction.

Two days after the first extraction the left.lower third molar was removed and the postoperative hemorrhage was again easily controlled with dried beef globulin substance applied locally in the same manner. The use of other agents was not attempted in this instance. The socket was completely healed in seven days.

* All dental extractions were performed by the Oral Surgery Department of the Boston City Hospital under local novocaine anesthesia. All teeth extracted had extensive caries of the crowns but the gums appeared healthy and the roots were solidly imbedded. 


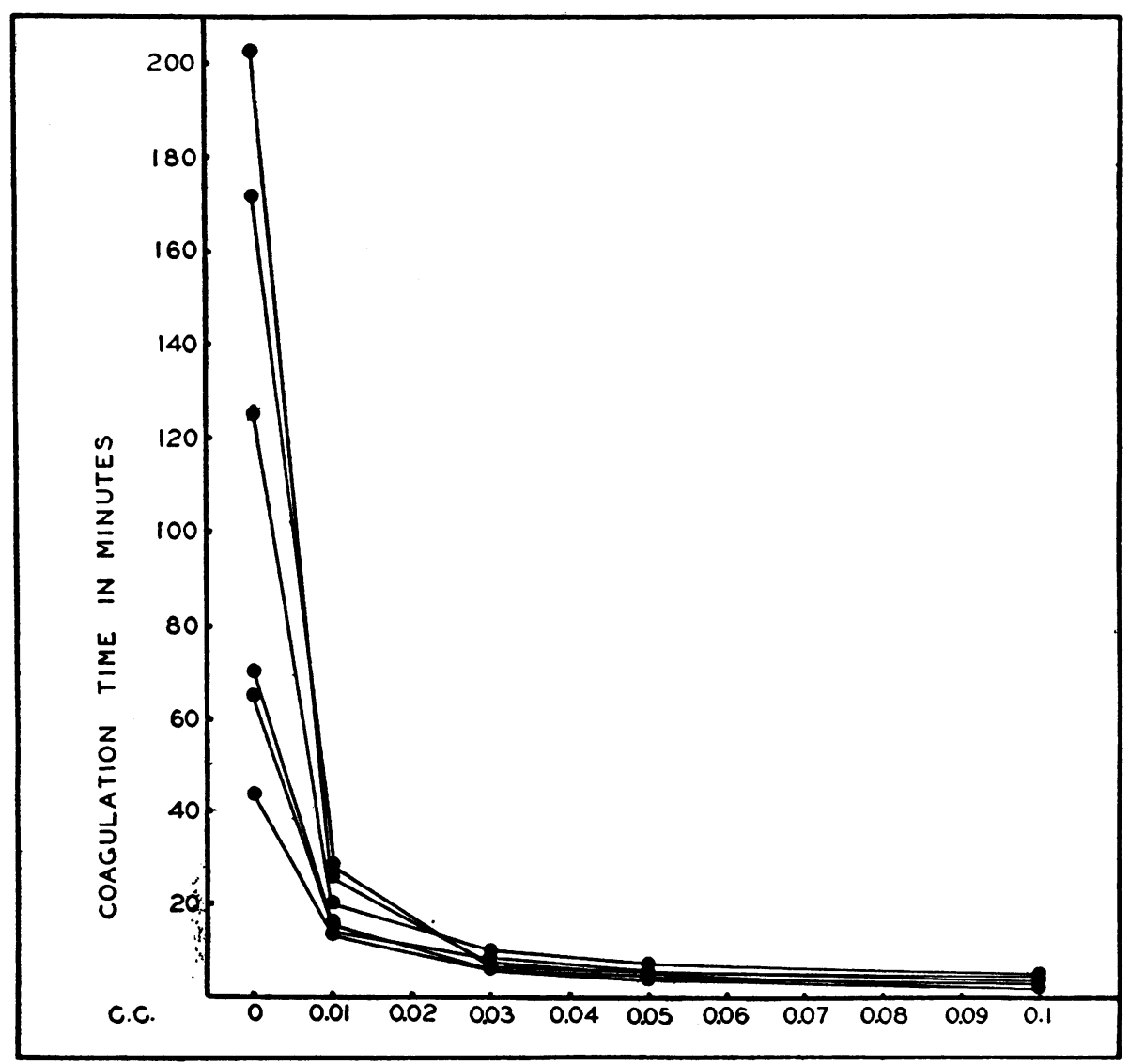

Fig. 2. Quantitative Effect of a Saline Suspension of Beef Globulin Substance on the Coagulation Time of the Blood from Six Hemophilic Subjects in Vitro

Case II. (Coagulation time from 40 to 60 minutes.) Following the extraction of the right upper third molar in June, 1936, the wound bled continuously for 16 days. Hemorrhage could not be controlled by the local application of placental extract, and three $500 \mathrm{cc}$. blood transfusions were required to keep the hemoglobin from becoming dangerously low.

Six months later the lower left second molar was removed and again bleeding followed. More than ordinary trauma was acquired during this extraction. The tooth socket was successively packed with liquid preparations of epinephrine, thromboplastin, cephalin, and a saline suspension of beef globulin substance without hemostasis. Dried beef globulin substance was then applied with the usual technique. A firm clot formed in three minutes and hemorrhage ceased. Again the pack was voluntarily removed every 24 hours to permit irrigation of the dental socket. Each time bleeding recommenced but was immediately controlled by repacking with dried beef globulin substance until healing was complete on the eighth day.

Subsequently three upper molar teeth were removed at two operations and the postoperative hemorrhage was always readily controlled with the local application of dried beef globulin substance. In each instance the dental sockets healed in seven days.

Case III. (Coagulation time from 150 to 180 minutes.) This patient was admitted to the hospital because of a left compression sciatic neuritis resulting from a hematoma. A short leg plaster cast was made to maintain proper position of the foot. While bivalving the cast the base of the fifth toe was accidentally cut. Bleeding was profuse for one hour but the local application of dried beef globulin substance produced immediate hemostasis. The dressing and clot were disturbed daily in removing the cast for physiotherapy and bleeding started again but each time it was readily controlled by repetition of the above procedure. The wound was cleanly healed after six days.

Case IV. (Coagulation time from 60 to 90 minutes.) Following the extraction of a tooth five years previously this patient bled continuously for 13 days and required three "large" blood transfusions.

On December 8, 1937, the right lower first, second, and third molar teeth were extracted resulting in extensive 
gum laceration and bone trauma. Hemorrhage could not be checked with the use of thromboplastin or epinephrine packs. The direct application of dried beef globulin substance on a saline moistened pack arrested the hemorrhage in six minutes. Mild pressure to hold the pack and clot in place was obtained by a gauze "bite." In this case, however, the pack would only hold for 8 to 24 hours and then bleeding would recur because of loosening of the clot at the edge of the wound. However, removal of the clot and repacking with dried beef globulin substance produced complete hemostasis for several hours at a time. After 11 days the alveolar ridge was completely healed.

Case $V$. (Coagulation time from 60 to 70 minutes.) The patient sustained two deep jagged wounds each 5 $\mathrm{cm}$. in length over the thenar eminence from broken glass. The wound was sutured but the formation of a large hematoma required their removal. Bleeding was continuous for $\mathbf{1 0}$ days in spite of the local application of thromboplastin and placental extract and two $500 \mathrm{cc}$. blood transfusions were required. A few days after the last transfusion the patient was admitted to the hospital. The hemoglobin was 8.58 grams per 100 cc. of blood, and there was a continuous ooze of blood from the wounds. After the removal of all clots and debris, dried beef globulin substance was applied locally in the usual manner and hemorrhage ceased immediately. There was no recurrence of bleeding and when the dressings were removed six days later the wounds were completely healed.

\section{DISCUSSION AND SUMMARY}

Globulin substance prepared by isoelectric precipitation at $\mathrm{pH} 6.0$ from citrated cellular-free beef plasma has properties similar to those discussed previously (1) for normal human globulin substance. Beef globulin substance is thermolabile, insoluble in water, partially soluble in physiological saline solution, and possesses marked clot accelerating powers for hemophilic blood in vitro. Its oral administration to two adult hemophilic subjects was without effect on the coagulation time of the blood with the doses employed. The parenteral administration of beef globulin substance has not as yet been attempted.

The present observations indicate that beef globulin substance in dried form is an effective local hemostatic in hemophilia. Its effectiveness seems to be due primarily to the rapid formation of a firm fibrin clot at the point of application. Twelve hemorrhagic episodes, in five hemophilic subjects, including nine dental extractions, a laceration through the scalp, and wounds of a toe and hand, have been treated. There was no change in the hemoglobin values as a result of the dental extractions described. There were no wound infections.

Some of the success of this local therapy may be attributed to factors other than the clot accelerating properties of beef globulin substance for hemophilic blood. All of the patients were extremely cooperative adults and were hospitalized. The patients whose teeth were extracted tolerated liquid diets until healing was complete. Care was used in applying the packs taking into account the size, shape, and depth of the wound and in each instance the active agent was brought directly in contact with the original site of hemorrhage.

The failure of liquid preparations to produce hemostasis may be explained by the fact that the clot accelerating material was greatly diluted or washed away before the several minutes necessary for clot formation elapsed. Dried globulin substance, however, could be greatly concentrated at the bleeding point and maintained there until a firm fibrin clot was formed. The brief time required for the formation of this clot was quite comparable to the time required for the patient's blood to clot in a test tube after the addition of a saline suspension of globulin substance.

Birch (3) in a recent review of the causes of death in 113 hemophilic individuals found that 20 per cent died from hemorrhage resulting from small accidental cuts and that another 20 per cent died from hemorrhage caused by minor external surgical procedures including dental extractions. The use of an effective local hemostatic may considerably decrease the danger attendant on external wounds in hemophilia.

\section{CONCLUSIONS}

1. Globulin substance, prepared from citrated cellular-free beef plasma by isoelectric precipitation at $\mathrm{pH} 6.0$, possesses properties similar to that prepared from normal human plasma, except that it has slightly greater clot accelerating properties for hemophilic blood in vitro.

2. The oral administration of beef globulin substance to adult hemophilics is without effect on the coagulation time of the blood in the doses employed. 
3. Beef globulin substance, applied locally in the form of a dry powder, is an effective hemostatic in hemophilia.

\section{BIBLIOGRAPHY}

1. Patek, Arthur J., Jr., and Taylor, F. H. L., Hemophilia. II. Some properties of a substance obtained from normal human plasma effective in ac- celerating the coagulation of hemophilic blood. J. Clin. Invest., 1937, 16, 113.

2. Pohle, Frederick J., and Taylor, F. H. L., The coagulation defect in hemophilia. The effect in hemophilia of intramuscular administration of a globulin substance derived from normal human plasma. J. Clin. Invest., 1937, 16, 741.

3. Birch, Carroll La Fleur, Hemophilia. Clinical and Genetic Aspects. Univ. of Illinois Press, Urbana. 1937, p. 59. 